\title{
On Reading Comprehension Teaching for English Majors under Relevance Theory
}

\author{
Ping $\mathrm{He}^{1}$ \\ ${ }^{1}$ Foreign Languages College, Nanchang Normal University, Nanchang, China \\ Correspondence: Ping He, Foreign Languages, Nanchang Normal College, Nanchang, China. Tel: \\ 189-7086-6118. E-mail: 394938227@qq.com
}

Received: October 27, 2017 Accepted: December 2, 2017 Online Published: December 4, 2017

doi: 10.5539/elt.v11n1p46 URL: http://doi.org/10.5539/elt.v11n1p46

\begin{abstract}
Relevance Theory from the perspective of cognitive psychology argues that human communication is an ostensive-inferential process, and emphasizes the function of the optimal relevance for communication. In this sense, reading comprehension could be considered as a kind of communication in which the writer manifests his/her communication intention and the reader infers from the discourse codes; thereinto, the optimal relevance between the textual information and the reader's cognition is essential to the optimal contextual effects. This paper sets out to discuss the explanatory power of relevance theory to reading comprehension, with focus on differences among readers with different reading abilities in grasping the optimal relevance with the discourse and the cognitive context. Through the reading teaching experiment undertaken for a semester, the result shows that the application of relevance theory to reading by pinpointing reading purpose, setting reading tasks and constructing cognitive contexts benefits greatly to students' inference capability, hence their reading ability, which is also instructive for the teaching mode of reading courses.
\end{abstract}

Keywords: relevance theory, reading, teaching

\section{Introduction}

The advancement of teaching reading for college English majors is closely relevant to the improvement of the comprehensive quality of foreign language talents in China. At present, quite a few of universities or colleges in China still adopt the traditional teaching mode in reading courses which overemphasizes specific linguistic knowledge and grammar in the text. A great many of scholars and theorists have put forward a series of influential reading models, such as information processing model, psycholinguistic model, schematic view, and so forth. Since 1986 when Dan Sperber and Deirdre Wilson proposed Relevance Theory which provides a new perspective for pragmatic research for its exploration of human's communication mechanism on the basis of cognitive psychology, there have been some scholars (Carrel, 1989; Qinxia, 2001) pointing out that this theory enjoys powerful explanation in reading comprehension. They consider reading as a communication by means of written form between the writer and the reader, among whom Qinxia Shi emphasizes that "the process of reading comprehension is in fact a psychological process in which the reader tries the most to infer logically what the writer manifests most relevant to his/her cognition" (Qinxia, 2001). This paper attempts to explore the application of Relevance Theory to reading teaching based on the practice of reading teaching in a domestic college, and tries to put forward reading teaching strategies under the guidance of this theory in the hope of improving students' English reading skills and the reading teaching efficiency.

\section{Relevance Theory}

Relevance Theory firstly appears in Relevance: Communication and Cognition by Sperber and Wilson in 1986, according to which communication is a process of ostension-inference rather than one of encoding and decoding. Ostension refers to a "behavior which makes manifest an intention to make something manifest"(Sperber \&Wilson, 1986), and inference is "the process by which an assumption is accepted as true or probably true on the strength of the truth or probable truth of other assumptions" (ibid. :68). In this sense, communication involves the communicator's ostension and the audience's inference, just as, "ostensive-inferential communication consists in making manifest to an audience one's intention to make manifest a basic layer of information" (ibid.:54). In this communication process, it is the principle of relevance that makes manifest the intention behind the ostension. They point out that one common way of achieving relevance consists in 
providing the addressees with evidence which bears on some assumptions of his. They also hold that the comprehension process involves confirmation of assumptions in two quite different ways, one being that understanding a piece of ostensive behavior involves constructing and confirming a hypothesis about the communicator's informative intention while the other, the most relevant effect of ostension being to confirm some previous assumption of the audience, and the role of relevance in both communication and cognition reflects "on what is involved in constructing and confirming a hypothesis about the communicator's intentions; on what it is for a representation of the world to be modified and improved; and on the relation between the two"(ibid.:71).

The above arguments are rather instructive for reading comprehension, as reading is a kind of communication between the writer and the reader and in the process of communication, the text producer encodes his communicative information, while the receiver needs to decode, infer and comprehend what is encoded. Searching the most relevant effect from the reading texts is a key process in gaining the intentional information.

\section{Reading as a Form of Communication under Relevance Theory}

As has been stated above, reading is a kind of communication between the text producer and the reader. A piece of reading discourse can be regarded as a fixed system of encoded verbal symbols, which seems that the writer fails to involve the communication, while in fact in the encoding process the writer needs to consider the effect of making manifest his informative intention, that's to say, to make the ostensive stimulus attract the reader's attention by means of creating contextual effects like setting an ostensive title, the topic sentences, relevant information in context or culture. As for the reader, in this information processing, the reader needs to decode and infer the relevant information according to his previous linguistic knowledge, logic and cognitive context. If the text is targeted to those who share common culture with the writer, it will take less cognitive efforts. But it does mean that people with the same culture have no trouble in communication, for individuals' cognitive abilities vary due to different genetic, education and working background. Just as what Sperber and Wilson put, "While it is clear that members of the same linguistic community converge on the same language, and plausible that they converge on the same inferential abilities, the same is not true of their assumptions about the world" (ibid.:16). In this sense, for readers with a different culture from what the original text belongs to, this information process will be much tougher since the relevance in terms of cognitive and cultural contexts are less shared between the text producer and the audience.

So how to achieve the contextual effects in reading is a dominating issue before us. Some scholars have proposed several strategies to apply Relevance Theory to reading teaching. For example, narrow reading (Stephen, 1996), the choice-making of meanings of a word according to context and setting questions in relevance with the background information of the reading text (Qingxia, 2001), the reading mode of "title-questions-text" (Hu, 2008), pre-reading preparations (Jingshu, 2014), etc.. The above strategies put emphasis on the importance of context effects, which provides new perspectives and methods for reading teaching reform. Since readers' ability of exploring relevance between the discourse and context varies from person to person or group to group, the author carries on an experiment to testify the effect of application of Relevance Theory to reading teaching for students with different reading abilities.

\section{Experiment of Applying Relevance Theory to Reading Teaching}

\subsection{Research Purpose and Research Content}

The purpose of this experiment is to testify the effect of Relevance Theory in reading teaching, and to explore specific application of Relevance Theory to reading teaching. The research will be conducted by means of experiment and questionnaire survey to examine the function of Relevance Theory in reading teaching

\subsection{Research Questions}

Three questions are intended to be addressed:

a. What are the characteristics of the English majors' attitude towards reading and their reading abilities? b. In the aspects of attitude to reading and reading abilities, are there any changes after the experiment of applying Relevance Theory to reading teaching?

c. What' the function of Relevance Theory in reading?

\subsection{Participant Characteristics}

The participants in this experiment are the English majors in Class One and Class Two in Nanchang Normal College enrolled in 2015. Before the experiment, the teacher takes the advantage of questionnaire survey and reading comprehension test (the simulated test paper of TEM-4) to get the knowledge of the two classes' reading 
capabilities and attitude towards reading. The statistics of the questionnaires show that students in the two classes have no clear goals and the reading speed is quite low, sometimes failing to grasp the key information when encountering longer or more difficult texts as well as feeling the questions tricky to answer. As for the reasons for the reading problems, most of them believe that it is their low linguistic level, especially their limited vocabulary that influences their reading efficiency. In addition, the test scores turn out that Class One get 70.2 on average and Class Two 69.7, which shows that they are basically on the same level in reading level.

\subsection{Experimental Manipulations}

In order to get valid result, this experiment takes Class One as the experimental object and Class Two the controlling object. In the experimental class, Relevance Theory is applied to reading teaching and in the controlling class the reading teaching is conducted without the interference of Relevance Theory but in traditional reading models. This experiment undergoes for one school year, during which we firstly set the teaching object of inference and the optimal relevance in the English reading course for Class One, and design the action plan to guide the implementation process; then in reading teaching, we guide students to analyze the text before reading, set reading tasks, raise relevant questions, provide background information, and instruct them to infer from the context.

After the experiment, we test the reading ability of the two classes through examination and interviews before comparing and analyzing the testing results of the two classes. The examination result demonstrates that students in Class One get higher scores than Class Two on average. The result is shown as the following table:

Table 1. testing result

\begin{tabular}{lllll}
\hline class & mean & Standard Deviation & $\mathrm{T}$ & $\mathrm{P}$ \\
\hline experimental class & 73.4 & 9.214 & & \\
controlling class & 70.5 & 12.637 & -1.463 & 0.032 \\
\hline
\end{tabular}

As is shown in the table, the average score in the experimental class is 73.4 and 3.2 higher than its original score; while the controlling class gets 70.5 on average, just increasing by 0.8 . The standard deviation of the experimental class is 9.214 , lower than 12.637 of the controlling class, which indicates that the data points of the experimental class are closer to the mean than that of the controlling class, that's to say, the scores in the experimental class have a more concentrated dispersion than that of the controlling one. Besides, the T-value and P-value show the significance of the result, according to the statistics, the P-value in this experiment 0.032 is less than 0.05 , which reflects that the difference between the two classes is obvious and statistically significant. And through the questionnaire, students in Class One state that with background information and clearer reading objectives, they could read more quickly and understand more adequately while students in Class Two express their confusion when coming across some themes they are unfamiliar with.

\subsection{Discussion}

From the result, it can be concluded that the application of Relevance Theory to reading teaching helps to improve students' ability of exploring inference between discourse and the cognitive context. Background information in relevance with the reading text helps students to construct the relevance between their previous schema and new information, which calls for less cognitive efforts, resulting in speeding up their reading. In addition, the sense of analyzing the style of reading materials contributes to activating students' presumption of the intentional communication information and stimulating their inference potential. With questions raised by the teacher before reading, students will be more active in seeking answers from the text, and the optimal relevance of their cognitions could be stimulated as well. Likewise, after reading, if further questions are raised, students' inferential assumptions will get further examined, hence strengthening their comprehension or modifying their illogical inference.

\section{Application of Relevance Theory to Reading Teaching for English Majors}

It is known that Chinese students' knowledge about English culture is still quite limited since they are indoctrinated English grammars and lexical knowledge throughout their English learning period. As Relevance Theory addresses the ostensive-inferential communication and the contextual effects, which is quite instructive for reading, some specific ways of applying Relevance Theory to reading teaching are summarized as follows. 


\subsection{Enriching Students' English Culture Knowledge}

It is acknowledged that a certain amount of knowledge about the culture of English-speaking countries enables students to improve their reading skills. Although it's impossible for Chinese students to share the mutual-knowledge or common knowledge with English native speakers, yet more common knowledge facilitates inference of the writer's intentional information in reading. As is well-known, language is the carrier of culture, and English culture including biblical culture, history, custom, politics, education and so forth infiltrates in English language. With more mutual-knowledge about English culture, readers will pay less cognitive efforts. Schiffer (1972) argues that in communication if the hearer is to be sure of recovering the correct interpretation, the one intended by the speaker, every item of contextual information used in interpreting the utterance must be not only known by the speaker and hearer, but mutually known. Although Sperber and Wilson criticize that "the mutual-knowledge hypothesis as untenable" (1989: 21) and it's true that mutual knowledge cannot be activated exactly as the communicator intends, yet a certain amount of mutual knowledge could guarantee the success of communication. Especially for English learners, the cultural context mutually known between them and the writer helps to accelerate the inference of the intentional information in the text. In this sense, the mutual knowledge is the factor of relevance for the reader to infer from the discourse.

\subsection{Strengthening Students' Inferential Capability}

The way to strengthen students' inferential capability includes introducing Relevance Theory to them and instructing them to read by logic or inference. The purpose of introducing Relevance Theory lies in helping them to understand the nature of reading, that is, linguistic knowledge and grammar alone cannot guarantee successful comprehension of the reading text, and reading is rather a process of inference by cognitive contexts. "A context is a psychological construct, a subset of the hearer's assumptions about the world" (Sperber \& Wilson, 1986). Taking full advantage of context helps readers to infer the meaning of new words, expressions, and metaphors. That is reading by logic and inference. Take an expression "the best airline rates" for example. Almost all the students take the word "rates" as the meaning of "ratio" or "rank" for these two meanings are mostly common seen is the dictionary, but the exact meaning of "rates" in this expression is not as students guess, so the teacher needs to lead them to go back to the context with the expression. When they read the sentences "...most of the best airline rates just now are with a 21-day or a 30-day advance purchase. If you're traveling within 21 days the rates might tend to be a little higher", they find whether "ratio" or "rank" in explaining this word is illogical, and finally through the context and their cognition, they infer that the word "rates" should refer to "cost" or "price". Thus, context is a useful clue for readers to comprehend the exact meanings of words, expressions and discourses. In addition, in the process of communication, the writer often gives ostensions by setting an ostensive title, topic sentences, beginning paragraph, conclusions, which are facilitative in comprehending the main idea of the text.

\subsection{Setting Questions to Lead in Reading}

Before reading, through setting relevant questions, students' cognitive contexts will be partly stimulated, which helps to construct their perceptions to the text. The questions should not only involve the macroscopic information about the reading material including the background information, the writing style and so on, but also involve details about the text including meanings of linguistic units and information of specific events inside. From such two kinds of questions, readers could efficiently read alternately in top-down and bottom-up processing. "A top-down processing model states that information at the higher levels may influence processing at the lower levels. For instance, a sentence context may affect the identification of words within that sentence. Speaking more intuitively, we may say that a top-down model of processing is one in which one's expectations play a significant role" (Carroll, 2008) Bottom-up processing is defined as "that which proceeds from the lowest level to the highest level of processing in such a way that all of the lower levels of processing operate without influence from the higher levels"(ibid.: 56). In other words, top-down reading emphasizes processing information from the general to the specific. Through getting firstly the information of the general discourse structure, style, background information the introduction to the background information, the teacher picks up some low-frequency or difficult words and explains the meanings of them, which to some extent helps to save time in reading. However, it doesn't necessarily mean that all new words need to be explained; instead, the teacher could leave some words unexplained to give students the chance to guess the meaning in light of the context, hence cultivating their inference capability. According to schema theory, the comprehension of the text depends on interaction of these two information processing models, which can be defined as sorts of ostensions in the text, students could better infer the main idea and general meanings of some new words or the signified when encountering the specific linguistic information. Take the text of Unit 4A from New College English (Second Edition) Integrated Course 4 as an example, whose title is America as a Collage. Before reading, the 
teacher sets questions like "what kind of country is America?", "How much do you know about the history of America?", and then through free talk with students, which helps to stimulate their knowledge network, the teacher goes further to introduce the history, civilization and the structure of American society by means of which students get a general background information about the object discussed in this text-America. In addition, as students are about to read, the teacher sets a task for them, that's, trying to find the answer to the question "Is America going to decline like other great nations have before?", "What's the author's argument and do you agree with that?"; these questions are intended to help students clear reading purpose and focus on the key information, by which students could construct the optimal relevance between their cognitive context and the effective information in the text. As for bottom-up reading, it involves reading from the specific to the general, say, decoding specific linguistic units from words to sentences then to the whole text, which is often adopted in traditional teaching. This way, to some extent, ignores the function of the context, that is, the ostensions given by the writer, hence blocking readers' motives of inference, but it does not mean that this way of information processing comes into no effect. Take the above text for example again. Apart from the introduction to the background information, the teacher picks up some low-frequency or difficult words and explains the meanings of them, which to some extent helps to save time in reading. However, it doesn't necessarily mean that all new words need to be explained; instead, the teacher could leave some words unexplained to give students the chance to guess the meaning in light of the context, hence cultivating their inference capability. With the support of information about the lexical, syntactic, or discourse levels and the expectations of the whole text, readers' ability of handling information will be comprehensively strengthened. Hence, adopting such two reading strategies will definitely improve students' reading efficiency.

\subsection{Group Discussion}

This segment of group discussion in reading teaching is mainly carried out after reading. It aims at further activating students' cognition of the context. Since "individuals tend to be highly idiosyncratic and differences in life history necessarily lead to differences in memorized information" (Sperber\&Wilson, 1986:16), providing chances for students to exchange their ideas on the same text will broaden their ways of thinking and background information. At the same time, if the teacher raises some questions about the text after reading and encourages students to discuss in group, their comprehension will be enhanced as well. Besides, their inferential abilities, vocabulary, and oral English will be improved as a result. The teacher could even encourage students themselves to raise questions about the discourse, or write a composition around the same theme with the text, and they will be more active in seeking relevance about the information of the reading material. For instance, after reading the text titled The Trap of Credit Card, the teacher asks students to discuss in groups the motivation of the bank promoting credit cards to customers and the reasons for the customer being trapped in the credit card trap. After discussion, when reporters of each group exhibit the discussion results, the teacher will find that there exists some competition among groups in the aspects of opinions and linguistic expressions. It's obvious that students' ways of thinking have been broadened, which contributes further to their cognition and schema relevant to the topic of the text.

\section{Conclusion}

Up to date, various researches illustrate that Relevance Theory enjoys strong explanatory power for reading comprehension and instructive function for reading teaching. And the experiment undertaken in this study has testified the point. Relevance Theory is, of course, not the only facilitative theory to instruct reading and its teaching practice, but it provides an alternative perspective of reading as a form of communication and an ostensive-inferential process. Its emphasis on relevance based on cognitive contexts definitely inspires second-language learning and teaching. The text as a form of communication media is filled with ostensions manifested by the writer, so as long as readers try to adequately apply his previous schema knowledge to perceiving the new information, it will take less cognitive efforts in comprehending. Likewise, in reading courses, teachers need to introduce this theory to students for assisting them to master the nature of reading and try to equip them with enough background information for better contextual effects. What's more, adopting both top-down processing and bottom-up processing in reading by setting relevant questions enables students to read more effectively. To put it in a nutshell, reading comprehension teaching with the guidance of Relevance Theory has obvious advantages over the traditional teaching model which ignores the relevance between the discourse and the context. Meanwhile, there remain some further questions, like how to examine effectively students' inferential abilities? And how to construct the evaluation system of English reading teaching based on Relevance Theory? These are matters yet to be explored afterwards for promoting and refining the Relevance Theory-based English reading teaching. 


\section{Acknowledgments}

This work was supported by funding of the program: English Teaching and Research Innovation Team project and the Project of On Reading Comprehension Teaching for English Majors under Relevance Theory in Colloge.

\section{References}

David. W. C. (2008). Psychology of Language (5th ed.), Thomson Wadsworth.

Krashen, S. (1996). The Case for Narrow Reading. System, 1, 97-100. https://doi.org/10.1016/0346-251X(95)00054-N

Schiffer, S. (1972). Meaning. Oxford: Clarendon Press.

Sperber. D., \& Wilson, D. (1986). Relevance: Communication and Cognition. Oxford: Blackwell.

Ping, H. (2008). The Explanatory Power of Relevance Theory and Schema Theory to Reading Comprehension. Journal of Yangtze Normal University, 24(2), 138-141.

Jingshu, L. (2014). The Construction of English Reading Comprehension Teaching Model on the Basis of Relevance Theory. Journal of Shenyang Normal University (Social Science Edition), 38(2), 157-159.

Qingxia, S. (2001). Relevance Theory and Reading Comprehension Teaching. Foreign language Education, $22(3), 52-54$.

\section{Copyrights}

Copyright for this article is retained by the author(s), with first publication rights granted to the journal.

This is an open-access article distributed under the terms and conditions of the Creative Commons Attribution license (http://creativecommons.org/licenses/by/4.0/). 